\title{
P009: Epidemiology, microbiology and outcome of community-onset gram-negative bacteremias in a Greek University Hospital
}

\author{
A Gikas ${ }^{1 *}$, G Charitaki², M Gkika', P Karakosta², A Christidou² \\ From 2nd International Conference on Prevention and Infection Control (ICPIC 2013) \\ Geneva, Switzerland. 25-28 June 2013
}

\begin{abstract}
Introduction
According to the new classification community-onset bacteremias $(\mathrm{COB})$ are divided in healthcare-associated (HCAB) and community-acquired (CAB). The objective of this study was to apply this classification to a crosssectional study of patients with $\mathrm{COB}$ and to determine differences between the two groups in terms of epidemiology, treatment and outcome.
\end{abstract}

\section{Methods}

The study was conducted at the University Hospital of Heraklion, Greece, from March 2010 to November 2011. Patients were classified as HCAB or CABusing pre-defined selection criteria. Epidemiological, clinical and therapeutic characteristics, antimicrobial resistance and outcome were compared in both groups. The statistical analysis was performed using SPSS 19.0.

\section{Results}

Among 145 patients with gram-negative COB, 83 (57.2\%) had HCAB and 62 (42.8\%) had CAB. The frequency of malignant tumors, renal insufficiency and dementia was higher in patients with $\mathrm{HCAB}$ than with $\mathrm{CAB}$. In both groups Escherichia coli was the mostcommon causative agent but the prevalence of Klebsiella pneumoniae in $\mathrm{HCAB}$ was significantly higher than $\mathrm{CAB}$ (19.3\% vs. $4.8 \%)$. Patients with $\mathrm{HCAB}$ had higher Charlson score and higher Pitt bacteremia score, less frequent administration of appropriate empirical antibiotic treatment and higher probability of death than patients with $C A B$.

The antimicrobial resistance in $\mathrm{HCAB} \kappa \alpha \mathrm{I} C A B$ patients respectively, was found $27 / 83(32.5 \%)$ vs. $4 / 62(6.5 \%)$
$(\mathrm{P}<.001)$ to third-generation cephalosporins (3GC), 22/83 $(26.5 \%)$ vs. $7 / 62(11.3 \%)(\mathrm{P}=.021)$ to aminoglycosides, $29 / 83$ (34.9\%) vs. 9/62 (14.5\%) ( $\mathrm{P}=.005)$ to quinolones. Bacteria that produced ESBL were $16 / 76$ (21.1\%) vs. $2 / 59$ (3.4\%) $(\mathrm{P}=.003)$, and carbapenem-resistant were $10 / 83$ $(12.0 \%)$ vs. $2 / 62(3.2 \%)(\mathrm{P}=.056)$ in $\mathrm{HCAB} \kappa \alpha \iota \mathrm{CAB}$ patients respectively

\section{Conclusion}

There are significant differences in the severity of underlying diseases, causative pathogens, antibiotic resistance, outcome and treatment between the two groups. In our region 3GC, aminoglycosides or fluoroquinolones are proposed as appropriate empirical treatment for patients with $\mathrm{CAB}$, whereas in patients with $\mathrm{HCAB}$ carbapenems should be the initial therapy.

\section{Disclosure of interest}

None declared.

\section{Author details \\ ${ }^{1}$ Ind.Diseases Dpt-Inf Control Unit, University Hospiatal of Heraklion, Heraklion, Greece. ${ }^{2}$ Clinical Bacteriology, University Hospiatal of Heraklion, Heraklion, Greece.}

Published: 20 June 2013

doi:10.1186/2047-2994-2-S1-P9

Cite this article as: Gikas et al.: P009: Epidemiology, microbiology and outcome of community-onset gram-negative bacteremias in a Greek University Hospital. Antimicrobial Resistance and Infection Control 2013 2(Suppl 1):P9. 